General Mathematics Vol. 28, No. 1 (2020), 105-114

DOI: $10.2478 / \mathrm{gm}-2020-0008$

sciendo

\title{
Generalized Briot-Bouquet differential equation based on new differential operator with complex connections ${ }^{1}$
}

\author{
Rabha W. Ibrahim
}

\begin{abstract}
Inequality study is a magnificent field for investigating the geometric behaviors of analytic functions in the open unit disk calling the subordination and superordination. In this work, we aim to formulate a generalized differential-difference operator. We introduce a new class of analytic functions having the generalized operator. Some subordination results are included in the sequel.
\end{abstract}

2020 Mathematics Subject Classification: 30C45, 30C55.

Key words and phrases: Differential operator, univalent function, analytic function, subordination, unit disk, inequality.

\section{Introduction}

Inequalities in a complex domain indicate an important role in the theory of functions. They have applied to study the geometric interpolation of analytic functions in the open unit disk. In addition, they have employed to generalize different classes of analytic functions. Recently, Lupas [1,2] presented a combination of two important differential operators introduced by Ruscheweyh [13] and Sàlàgean [14] to indicate a set of inequalities and inclusions by utilizing the idea of subordination. The subordination inequalities are indicated a tool to define new classes of analytic functions in the open unit disk.

\footnotetext{
${ }^{1}$ Received 4 July, 2019

Accepted for publication (in revised form) 30 April, 2020
} 
Let $\Lambda$ be the class of analytic function formulated by

$$
f(z)=z+\sum_{n=2}^{\infty} a_{n} z^{n}, \quad z \in U=\{z:|z|<1\} .
$$

For a function $f \in \Lambda$, the Ruscheweyh derivative satisfies the formula

$$
R^{m} f(z)=z+\sum_{n=2}^{\infty} C_{m+n-1}^{m} a_{n} z^{n}
$$

where the term $C_{m+n-1}^{m}$ is the combination coefficients, the Sàlàgean operator achieves

$$
S^{m} f(z)=z+\sum_{n=2}^{\infty} n^{m} a_{n} z^{n}
$$

and the Lupas operator takes the formal

$$
L_{\alpha}^{m} f(z)=z+\sum_{n=2}^{\infty}\left[\alpha n^{m}+(1-\alpha) C_{m+n-1}^{m}\right] a_{n} z^{n}, \quad z \in U, \alpha \geq 0 .
$$

Recently, Ibrahim and Darus [6] presented the following operator

$$
\begin{aligned}
D_{\kappa}^{0} f(z) & =f(z) \\
D_{\kappa}^{1} f(z) & =z f^{\prime}(z)+\frac{\kappa}{2}(f(z)-f(-z)-2 z), \quad \kappa \in \mathbb{R} \\
\vdots & \\
D_{\kappa}^{m} f(z) & =D_{\kappa}\left(D_{\kappa}^{m-1} f(z)\right) \\
& =z+\sum_{n=2}^{\infty}\left[n+\frac{\kappa}{2}\left(1+(-1)^{n+1}\right)\right]^{m} a_{n} z^{n} .
\end{aligned}
$$

For $\kappa=0$, the operator is the Salagean's differential operator. Moreover, the second term of $D_{\kappa}^{m}$ is a modified Dunkl operator of complex variables [4], where $\kappa$ is called the Dunkl parameter.

In this note, we introduce a new operator combining $R^{m}$ and $D_{\kappa}^{m}$ as follows:

$$
\begin{aligned}
J_{\alpha, \kappa}^{m} f(z) & =(1-\alpha) R^{m} f(z)+\alpha D_{\kappa}^{m} f(z) \\
& =z+\sum_{n=2}^{\infty}\left[(1-\alpha) C_{m+n-1}^{m}+\alpha\left(n+\frac{\kappa}{2}\left(1+(-1)^{n+1}\right)\right)^{m}\right] a_{n} z^{n} .
\end{aligned}
$$

\section{Remark 1.1.}

- $m=0 \Longrightarrow J_{\alpha, k}^{0} f(z)=f(z)$;

- $\kappa=0 \Longrightarrow J_{\alpha, 0}^{m} f(z)=L_{\alpha}^{m} f(z)$; 
- $\alpha=0 \Longrightarrow J_{0, \kappa}^{m} f(z)=R^{m} f(z)$;

- $\alpha=1 \Longrightarrow J_{1, \kappa}^{m} f(z)=D_{\kappa}^{m} f(z)$;

- $\kappa=0, \alpha=1 \Longrightarrow J_{1,0}^{m} f(z)=S^{m} f(z)$.

Definition 1.2. Let $\epsilon \in[0,1), \alpha \geq 0, \kappa \geq 0$, and $m \in \mathbb{N}$. A function $f \in \Lambda$ is said to be in the set $\mathfrak{S}_{m}(\alpha, \kappa, \sigma)$ if and only if

$$
\frac{\left(J_{\alpha, \kappa}^{m} f(z)\right)^{\prime}}{J_{\alpha, \kappa}^{m} f(z)} \prec \sigma(z), \quad z \in U,
$$

where $\sigma$ is univalent function with a positive real part in $U$ satisfying $\sigma(0)=$ $1, \Re\left(\sigma^{\prime}(z)\right)>0$.

For functions $f$ and $g$ in $\Lambda$ we say that $f$ is subordinate to $g$, denoted by $f \prec g$, if there exists a Schwarz function $\omega \in U$ with $\omega(0)=0$ and $|\omega(z)|<1, z \in U$ so that $f(z)=g(\omega(z))$ for all $z \in U$ (see [12]). Evidently $f(z) \prec g(z)$ is equivalent to $f(0)=g(0)$ and $f(U) \subset g(U)$. Note that the class $\mathfrak{S}_{m}^{*}(\alpha, \lambda, \sigma)$ is a generalization of some classes of analytic functions. Moreover, this class is a specialist of Ma and Minda class [10] given as follows $\left(\mathfrak{S}^{*}(\sigma)\right)$ :

$$
\frac{z f^{\prime}(z)}{f(z)} \prec \sigma(z) .
$$

Moreover, when $\sigma(z)=1+\sin (z)$ and $m=0$, the class

$$
\frac{z f^{\prime}(z)}{f(z)} \prec 1+\sin (z)
$$

is studied by Cho et al. [3]. Our class is generalization of two classes given by Khatter et al [8] as follows:

$$
\frac{z f^{\prime}(z)}{f(z)} \prec \beta+(1-\beta) \sqrt{1+z}
$$

and

$$
\frac{z f^{\prime}(z)}{f(z)} \prec \beta+(1-\beta) e^{z},
$$

where $\beta=0$ introduces the class [11]

$$
\frac{z f^{\prime}(z)}{f(z)} \prec e^{z} .
$$

Kumar et al. [9] defined the class by using Bell numbers as follows:

$$
\frac{z f^{\prime}(z)}{f(z)} \prec e^{e^{z}-1}
$$


Lemma 1.3. If $\beta \in[0,1], z \in U$ then each function of the form

- $\sigma(z)=\beta+(1-\beta) \sqrt{1+z}$,

- $\sigma(z)=\beta+(1-\beta) e^{z}$,

- $\sigma(z)=\beta+(1-\beta)(1+\sin (z))$,

- $\sigma(z)=\beta+(1-\beta) e^{e^{z}-1}$,

has the upper and lower bound for all $r \in(0,1), \theta \in[0,2 \pi)$ as follows:

$$
\min _{|z|=r} \Re(\sigma(z))=\sigma(-r)=\min _{|z|=r}|\sigma(\xi)|
$$

and

$$
\max _{|z|=r} \Re(\sigma(z))=\sigma(r)=\max _{|z|=r}|\sigma(z)| .
$$

Proof. The first and second type can be located in [8]. We only need to prove the third type. For $\beta=0$, we have the function $\sigma(z)=1+\sin (z)$ (see [3]). It is clear that

$$
\sin (z)=\sin \left(r e^{i \theta}\right)=\sin (r \cos (\theta)) \cosh (r \sin (\theta))+i \cos (r \cos (\theta)) \sinh (r \sin (\theta))
$$

therefore, we have

$$
\Re(\sigma(z))=1+\sin (r \cos (\theta)) \cosh (r \sin (\theta)) .
$$

Consequently, by taking $r \rightarrow 0$, we obtain

$$
\min _{|z|=r} \Re(\sigma(z))=1-\sin (r)=\min _{|z|=r}|\sigma(z)|=1 .
$$

Moreover, we have

$\left|\sin \left(r e^{i \theta}\right)\right|^{2}=\cos ^{2}(r \cos \theta) \sinh 2(r \sin \theta)+\sin ^{2} 2(r \cos \theta) \cosh 2(r \sin r) \leq \sinh ^{2}(r) ;$

thus, this yields

$$
\max _{|z|=r} \Re(\sigma(z))=1+\sin (r)=\max _{|z|=r}|\sigma(z)| \leq 1+\sinh ^{2}(r) .
$$

Extend the above result, for $\beta>0$, we have

$$
\min _{|z|=r} \Re(\sigma(z))=\beta+(1-\beta)(1-\sin (r))=\min _{|z|=r}|\sigma(z)|=1,
$$

and

$$
\max _{|z|=r} \Re(\sigma(z))=\beta+(1-\beta)(1+\sin (r))=\max _{|z|=r}|\sigma(z)| \leq \beta+(1-\beta)\left(1+\sinh ^{2}(r)\right) .
$$

Similarly for the last assertion.

The next result can be found in [12] 
Lemma 1.4. If $\tau>0$ and $\sigma \in \mathfrak{H}[1, n]$, then there are constants $\wp>0$ and $v>0$ with $v=v(\wp, \tau, n)$ so that

$$
\sigma(z)+\tau z \sigma^{\prime}(z) \prec\left[\frac{1+z}{1-z}\right]^{v} \Rightarrow \sigma(z) \prec\left[\frac{1+z}{1-z}\right]^{\wp} .
$$

Lemma 1.5. Let $\varphi(z)$ be a convex function in $U$ and $h(z)=\varphi(z)+n v\left(z \varphi^{\prime}(z)\right)$ for $v>0$ and $n$ is a positive integer. If $\varrho \in \mathfrak{H}[\varphi(0), n]$, and

$$
\varrho(z)+v z \varrho^{\prime}(z) \prec h(z), \quad z \in U,
$$

then

$$
\varrho(z) \prec \varphi(z)
$$

and this result is sharp.

\section{Results}

In this section, we deal with the class $\mathfrak{S}_{m}^{*}(\alpha, \kappa, \sigma)$ for special types of $\sigma(z)$ given in Lemma 1.3.

Theorem 2.1. The class $\mathfrak{S}_{m}^{*}(\alpha, \kappa, \sigma)$ achieves the following inclusion:

$$
\mathfrak{S}_{m}^{*}(\alpha, \kappa, \sigma) \subset \mathfrak{S}_{m}^{*}(\alpha, \kappa, \gamma) \subset \mathfrak{S}_{m}^{*}(\alpha, \kappa),
$$

where $\sigma$ is one of the type in Lemma 1.3 and

$$
\begin{gathered}
\mathfrak{S}_{m}^{*}(\alpha, \kappa, \gamma):=\left\{f \in \Lambda \Re\left(\frac{\left.z\left(J_{\alpha, \kappa}^{m} f(z)\right)\right)^{\prime}}{J_{\alpha, \kappa}^{m} f(z)}\right)>\gamma\right\} ; \\
\mathfrak{S}_{m}^{*}(\alpha, \kappa):=\left\{f \in \Lambda \Re\left(\frac{\left.z\left(J_{\alpha, \kappa}^{m} f(z)\right)\right)^{\prime}}{J_{\alpha, \kappa}^{m} f(z)}\right)>0\right\} .
\end{gathered}
$$

Proof. Let $\curlyvee \in \mathfrak{S}_{m}^{*}(\alpha, \kappa, \sigma)$ and let $\sigma(z)=\beta+(1-\beta) \sqrt{1+z}$, then we have the inequality

$$
\frac{\left.z\left(J_{\alpha, \kappa}^{m} f(z)\right)\right)^{\prime}}{J_{\alpha, k}^{m} f(z)} \prec \beta+(1-\beta) \sqrt{1+z}, \quad z \in U .
$$

In view of Lemma 1.3, we obtain

$$
\min _{|z|=1^{-}} \Re(\beta+(1-\beta) \sqrt{1+z})<\Re\left(\frac{\left.z\left(J_{\alpha, k}^{m} f(z)\right)\right)^{\prime}}{J_{\alpha, k}^{m} f(z)}\right)<\max _{|z|=1^{+}} \Re(\beta+(1-\beta) \sqrt{1+z}),
$$

which yields

$$
\left.\beta<\Re\left(\operatorname{dfracz}\left(J_{\alpha, \kappa}^{m} f(z)\right)\right)^{\prime} J_{\alpha, \kappa}^{m} f(z)\right)<\beta+(1-\beta) \sqrt{2} .
$$


Hence, we have

$$
\Re\left(\frac{\left.z\left(J_{\alpha, \kappa}^{m} f(z)\right)\right)^{\prime}}{J_{\alpha, \kappa}^{m} f(z)}\right)>\beta:=\gamma \geq 0,
$$

consequently, we get the requested result. Consider $\sigma(z)=\beta+(1-\beta) e^{z}$, the we have

$$
\min _{|z|=1} \Re\left(\beta+(1-\beta) e^{z}\right)<\Re\left(\frac{\left.z\left(J_{\alpha, \kappa}^{m} f(z)\right)\right)^{\prime}}{J_{\alpha, \kappa}^{m} f(z)}\right)<\max _{|z|=1} \Re\left(\beta+(1-\beta) e^{z}\right),
$$

which implies

$$
\left(\beta+(1-\beta) \frac{1}{e}\right)<\Re\left(\frac{\left.z\left(J_{\alpha, \kappa}^{m} f(z)\right)\right)^{\prime}}{J_{\alpha, \kappa}^{m} f(z)}\right)<(\beta+(1-\beta) e),
$$

that is

$$
\Re\left(\frac{\left.z\left(J_{\alpha, \kappa}^{m} f(z)\right)\right)^{\prime}}{J_{\alpha, \kappa}^{m} f(z)}\right)>\left(\beta+(1-\beta) \frac{1}{e}\right):=\gamma \geq 0 .
$$

Similarly, by letting $\sigma(z)=\beta+(1-\beta)(1+\sin (z))$ then we have

$$
\min _{|z|=1} \Re(\beta+(1-\beta)(1+\sin (z)))<\Re\left(\frac{\left.z\left(J_{\alpha, \kappa}^{m} f(z)\right)\right)^{\prime}}{J_{\alpha, \kappa}^{m} f(z)}\right)<\max _{|z|=1} \Re(\beta+(1-\beta)(1+\sin (z))),
$$

which leads to

$$
(\beta+0.158(1-\beta))<\Re\left(\frac{\left.z\left(J_{\alpha, \kappa}^{m} f(z)\right)\right)^{\prime}}{J_{\alpha, \kappa}^{m} f(z)}\right)<(\beta+1.841(1-\beta)),
$$

this brings the inequality

$$
\Re\left(\frac{\left.z\left(J_{\alpha, \kappa}^{m} f(z)\right)\right)^{\prime}}{J_{\alpha, \kappa}^{m} f(z)}\right)>(\beta+0.158(1-\beta)):=\gamma \geq 0 .
$$

Remark 2.2. In Theorem 2.1,

- $m=0, \beta=0, \sigma(z)=1+\sin z \Longrightarrow[3]$

- $m=0 \Longrightarrow[8]$

- $m=0, \beta=0, \sigma(z)=e^{z} \Longrightarrow[7]$

- $m=0, \beta=0, \sigma(z)=\sqrt{1+z} \Longrightarrow[7]$.

Theorem 2.3. The class $\mathfrak{S}_{m}^{*}(\alpha, \kappa, \sigma)$ achieves the following inclusion:

$$
\mathfrak{S}_{m}^{*}(\alpha, \kappa, \sigma) \subset \mathfrak{M}_{m}(\alpha, \kappa, \gamma):=\left\{f \in \Lambda \Re\left(\frac{\left.z\left(J_{\alpha, \kappa}^{m} f(z)\right)\right)^{\prime}}{J_{\alpha, \kappa}^{m} f(z)}\right)<\gamma, \gamma>1\right\} .
$$

where $\sigma$ is given in Lemma 1.3. 
The set $\mathfrak{M}_{m}(\alpha, \lambda, \gamma)$ is a generalization of the set

$$
\mathfrak{M}(\gamma):=\left\{f \in \Lambda \Re\left(\frac{\left.z\left(J_{\alpha, \lambda}^{m} f(z)\right)\right)^{\prime}}{J_{\alpha, \lambda}^{m} f(z)}\right)<\gamma, \gamma>1\right\}
$$

given by Uralegaddi et al. [15].

Proof. Let $f \in \mathfrak{S}_{m}^{*}(\alpha, \lambda, \sigma)$, where $\sigma$ is given in Lemma 1.3. By the proof of Theorem 2.1, we have

$$
\begin{gathered}
\Re\left(\frac{\left.z\left(J_{\alpha, \lambda}^{m} f(z)\right)\right)^{\prime}}{J_{\alpha, \lambda}^{m} f(z)}\right)<\beta+(1-\beta) \sqrt{2}:=\gamma, \\
\Re\left(\frac{\left.z\left(J_{\alpha, \lambda}^{m} f(z)\right)\right)^{\prime}}{J_{\alpha, \lambda}^{m} f(z)}\right)<\beta+(1-\beta) e:=\gamma
\end{gathered}
$$

and

$$
\Re\left(\frac{\left.z\left(J_{\alpha, \kappa}^{m} f(z)\right)\right)^{\prime}}{J_{\alpha, \kappa}^{m} f(z)}\right)<(\beta+1.841(1-\beta)):=\gamma,
$$

Hence, $f \in \mathfrak{M}_{m}(\alpha, f(z), \gamma)$, where the value of $\gamma$ based on the function $\sigma$, which completes the proof.

\section{Remark 2.4. In Theorem 2.3,}

- $m=0, \beta=0, \sigma(z)=1+\sin z \Longrightarrow[3]$

- $m=0, \sigma(z)=\beta+(1-\beta) e^{z} \Longrightarrow[8]$, Theorem 2.5;

- $m=0, \sigma(z)=\beta+(1-\beta)(\sqrt{1+z}) \Longrightarrow[8]$, Theorem 2.6.

- $m=0, \beta=0, \sigma(z)=(\sqrt{1+z}) \Longrightarrow[8]$, Corollary 2.7.

Theorem 2.5. If $f \in \Lambda$ satisfies the subordination

$$
\left(\frac{z\left(J_{\alpha, \kappa}^{m} f(z)\right)^{\prime}}{J_{\alpha, \kappa}^{m} f(z)}\right)\left(2+\frac{z\left(J_{\alpha, \kappa}^{m} f(z)\right)^{\prime \prime}}{\left(J_{\alpha, \kappa}^{m} f(z)\right)^{\prime}}-\frac{z\left(J_{\alpha, \kappa}^{m} f(z)\right)^{\prime}}{J_{\alpha, \kappa}^{m} f(z)}\right) \prec\left[\frac{1+z}{1-z}\right]^{\tau}
$$

then $f \in \mathfrak{S}_{m}^{*}(\alpha, \kappa, \sigma)$, where $\sigma(z)=\left[\frac{1+z}{1-z}\right]^{\wp}$ for $\wp>0, \tau>0$.

Proof. We aim to apply Lemma 1.4, a calculation implies that

$$
\begin{aligned}
& \left(\frac{z\left(J_{\alpha, \kappa}^{m} f(z)\right)^{\prime}}{J_{\alpha, \kappa}^{m} f(z)}\right)+z\left(\frac{z\left(J_{\alpha, \kappa}^{m} f(z)\right)^{\prime}}{J_{\alpha, \kappa}^{m} f(z)}\right)^{\prime} \\
& =\left(\frac{z\left(J_{\alpha, \kappa}^{m} f(z)\right)^{\prime}}{J_{\alpha, \kappa}^{m} f(z)}\right)\left(2+\frac{z\left(J_{\alpha, \kappa}^{m} f(z)\right)^{\prime \prime}}{\left(J_{\alpha, \kappa}^{m} f(z)\right)^{\prime}}-\frac{z\left(J_{\alpha, \kappa}^{m} f(z)\right)^{\prime}}{J_{\alpha, \kappa}^{m} f(z)}\right) \\
& \prec\left[\frac{1+z}{1-z}\right]^{\tau} .
\end{aligned}
$$


Thus, in view of Lemma 1.4, we have

$$
\left(\frac{z\left(J_{\alpha, k}^{m} f(z)\right)^{\prime}}{J_{\alpha, k}^{m} f(z)}\right) \prec\left[\frac{1+z}{1-z}\right]^{\wp}:=\sigma(z),
$$

which implies that $f \in \mathfrak{S}_{m}^{*}(\alpha, \kappa, \sigma)$.

Theorem 2.6. Let $\varphi$ be a convex function such that $\varphi(0)=0$ and let $\hbar$ be the function

$$
\hbar(z)=\varphi(z)+\frac{z}{1-\ell} \varphi^{\prime}(z), \quad z \in U, \ell \in(0,1) .
$$

If for a function $f \in \Lambda$ satisfies the subordination

$$
\left(\frac{z}{J_{\alpha, \kappa}^{m+1} f(z)}\right)^{\ell} \frac{J_{\alpha, \kappa}^{m} f(z)}{1-\ell}\left(\frac{\left(J_{\alpha, \kappa}^{m+1} f(z)\right)^{\prime}}{J_{\alpha, \kappa}^{m+1} f(z)}-\ell \frac{\left(J_{\alpha, \kappa}^{m} f(z)\right)^{\prime}}{J_{\alpha, \kappa}^{m} f(z)}\right) \prec \hbar(z)
$$

then

$$
\left(\frac{J_{\alpha, \kappa}^{m+1} f(z)}{z}\right)\left(\frac{z}{J_{\alpha, \kappa}^{m+1} f(z)}\right)^{\ell} \prec \varphi(z), \quad z \in U .
$$

The outcome is sharp.

Proof. We aim to apply Lemma 1.5. Let

$$
\varrho(z)=\left(\frac{J_{\alpha, \kappa}^{m+1} f(z)}{z}\right)\left(\frac{z}{J_{\alpha, \kappa}^{m+1} f(z)}\right)^{\ell} .
$$

A differentiation implies that

$$
\left(\frac{\xi}{J_{\alpha, \kappa}^{m+1} f(z)}\right)^{\ell} \frac{J_{\alpha, \kappa}^{m} f(z)}{1-\ell}\left(\frac{\left(J_{\alpha, \kappa}^{m+1} f(z)\right)^{\prime}}{J_{\alpha, \kappa}^{m+1} f(z)}-\ell \frac{\left.\left(J_{\alpha, \kappa}^{m}\right) f(z)\right)^{\prime}}{J_{\alpha, \kappa}^{m} f(z)}\right)=\varrho(z)+\left(\frac{1}{1-\ell}\right) z \varrho^{\prime}(z)
$$

Thus, by the assumption, we have

$$
\varrho(z)+\left(\frac{1}{1-\ell}\right) z Q^{\prime}(z) \prec \hbar(z)=\varphi(z)+\frac{z}{1-\ell} \varphi^{\prime}(z), \quad z \in U .
$$

By employing Lemma 1.5 yields $\varrho(z) \prec \hbar(z)$, which means

$$
\left(\frac{J_{\alpha, \kappa}^{m+1} f(z)}{z}\right)\left(\frac{z}{J_{\alpha, \kappa}^{m+1} f(z)}\right)^{\ell} \prec \varphi(z), \quad z \in U .
$$

This result is sharp.

Remark 2.7. In Theorem 2.5, $\lambda=0 \Longrightarrow$ [2] Theorem 2.14. 
Example 2.8. Consider the following data:

$$
f(z)=\frac{z}{1-z}, \quad \alpha=\kappa=m=1
$$

then, we have

$$
J_{\alpha, k}^{m} f(z)=z+2 z^{2}+4 z^{3}+4 z^{4}+6 z^{5}+6 z^{6}+O\left(z^{7}\right),|z|<1
$$

and

$$
P(z)=\frac{\left.z\left(J_{\alpha, \lambda}^{m} f(z)\right)\right)^{\prime}}{J_{\alpha, \lambda}^{m} f(z)}=1+2^{z}+4 z^{2}-4 z^{3}+8 z^{4}+2 z^{5}+O\left(z^{6}\right),|z|<1 .
$$

By using Wolram Alpha, we have

$$
1 \leq \Re(P(z)) \leq 1.83929: \gamma, \beta \rightarrow 0 .
$$

Moreover, the operator $J_{\alpha, \lambda}^{m} f(z)$ is univalent for $-0.0001<\kappa \leq 0.0001$, where (Biberbach Conjecture [5])

$$
J_{\alpha, \lambda}^{m} f(z)=z+2 z^{2}+3.000 z^{3}+4 z^{4}+5.000 z^{5}+6 z^{6}+O\left(z^{7}\right),\left|a_{n}\right| \leq n .
$$

Then in view of Theorem 2.3, we have $J_{\alpha, \lambda}^{m} f(z) \in \mathfrak{M}_{m}(\alpha, \kappa, \gamma)$, with $\sigma(z)=\beta+$ $(1-\beta)(1+\sin (z))$. The roots of $P(z)$ in $U$

$$
R_{1,2}=-0.41 \pm 0.32 i, R_{3,4}=-0.49 \pm 0.46 i, R_{5,6}=-0.11 \pm 0.47 i, R_{7,8} \approx \pm 1 .
$$

\section{Conclusion}

This study indicated a class of differential subordination inequalities using a new differential operator of complex connections. Some inequalities containing the subordination concept are extended recent works.

\section{Acknowledgment}

The author would like to express their fully thanks to the respected reviewers for the deep comments to improve our paper.

\section{References}

[1] A. A. Lupas, On special differential subordinations using Salagean and Ruscheweyh operators, Math. Inequal. Appl., vol. 12, no. 4, 2009, 781-790.

[2] A. A. Lupas, Some differential subordinations using Ruscheweyh derivative and Salaagean operator, Advances in Difference Equations, vol. 2013, no. 150, 2013, $1-12$. 
[3] N. E. Cho, et al., Radius problems for starlike functions associated with the sine function, Bull. Iran. Math. Soc., vol.45, no.213, 2019, 1-20.

[4] C. F. Dunkl, Differential-difference operators associated with reflections groups, Trans. Am. Math. Soc., vol. 311, 1989, 167-183.

[5] P. Duren, Univalent Functions, Grundlehren der mathematischen Wissenschaften; 259 Springer-Verlag New York Inc., 1983.

[6] R. W. Ibrahim, M. Darus, Subordination inequalities of a new Salagean-difference operator, International Journal of Mathematics and Computer Science, vol. 14, no. 3, 2019, 573-582.

[7] S. Kanas, A. Wisniowska, Conic domains and starlike functions, Rev. Roum. Math. Pures Appl., vol. 45, no. 4, 2000, 647-657.

[8] K. Khatter, et al., Starlike functions associated with exponential function and the lemniscate of Bernoulli, Revista de la Real Academia de Ciencias Exactas, Serie A. Matematicas, vol. 113, no. 1, 2019, 233-253.

[9] V. Kumar, et al., Sharp coefficient bounds for starlike functions associated with the Bell numbers, Math. Slovaca, vol. 69, 2019, 1053-1064.

[10] W. C. Ma, D. Minda, A unified treatment of some special classes of univalent functions, In Proceedings of the Conference on Complex Analysis, Tianjin, China, vol. 19, no. 23, 1992, 1-13.

[11] R. Mendiratta, S. Nagpal., V.Ravichandran, On a subclass of strongly starlike functions associated with exponential function, Bull. Malays. Math. Sci. Soc., vol. 38, 2015, 365-386.

[12] S. S. Miller, P. T. Mocanu, Differential subordinations: theory and applications, CRC Press, 2000.

[13] S. Ruscheweyh, New criteria for univalent functions, Proc. Amet. Math. Soc., vol. 49, 1975, 109-115.

[14] G. St. Sălăgean, Subclasses of univalent functions, Lecture Notes in Math., Springer Verlag, Berlin, vol. 1013, 1983, 362-372.

[15] B. A. Uralegaddi, et al., Univalent functions with positive coefficients, Tamkang J. Math., vol. 25, no. 3, 1994, 225-230.

\section{Rabha W. Ibrahim}

Ton Duc Thang University

Faculty of Mathematics \& Statistics

Informetrics Research Group

Ho Chi Minh City, Vietnam

e-mail: rabhaibrahim@tdtu.edu.vn, rabhaibrahim@yahoo.com 